\begin{tabular}{|c|l|}
\hline Title & Real-time evolution method and its application to the 3a cluster system \\
\hline Author(s) & Imai, R.; Tada, T.; Kimura, M. \\
\hline Citation & $\begin{array}{l}\text { Physical Review C, 99(6), 064327 } \\
\text { https://doi.org/10.1103/PhysRevC.99.064327 }\end{array}$ \\
\hline Issue Date & 2019-06-24 \\
\hline Doc URL & http://hdl.handle.net/2115/75705 \\
\hline Rights & ○2019A merican Physical Society \\
\hline Type & article \\
\hline File Information & PhysRevC.99.064327.pdf \\
\hline
\end{tabular}

Instructions for use 


\title{
Real-time evolution method and its application to the $3 \alpha$ cluster system
}

\author{
R. Imai, ${ }^{1}$ T. Tada, ${ }^{1}$ and M. Kimura ${ }^{1,2, *}$ \\ ${ }^{1}$ Department of Physics, Hokkaido University, 060-0810 Sapporo, Japan \\ ${ }^{2}$ Nuclear Reaction Data Centre, Faculty of Science, Hokkaido University, 060-0810 Sapporo, Japan
}

(Received 10 February 2018; revised manuscript received 12 May 2019; published 24 June 2019)

\begin{abstract}
A theoretical method is proposed to describe the ground and excited cluster states of atomic nuclei. The method makes use of the equation of motion of the Gaussian wave packets to generate the basis wave functions incorporating various cluster configurations. The generated basis wave functions are superposed to diagonalize the Hamiltonian. This method is regarded as the generator coordinate method which uses the real time as the generator coordinate. As a application of our proposed method, we present the benchmark calculation for the $3 \alpha$ system. We show that our proposed method works efficiently and yields the results consistent with or better than the other cluster models. We have also discussed briefly the structure of the excited $0^{+}$and $1^{-}$states.
\end{abstract}

DOI: 10.1103/PhysRevC.99.064327

\section{INTRODUCTION}

Since the last four decades, it has been known that the Hoyle state (the $0_{2}^{+}$state of ${ }^{12} \mathrm{C}$ ) is a dilute gas-like $\alpha$ cluster state dominated by the $s$ wave [1-8]. In the beginning of this century, it was claimed that the Hoyle state can be regarded as a Bose-Einstein condensate of $\alpha$ particles [9-11]. These findings triggered the initiation of many studies on the structure of ${ }^{12} \mathrm{C}$. The idea of the $\alpha$-particle condensate has been extended to other excited states above the Hoyle state. Namely, the $2^{+}$state at $10.03 \mathrm{MeV}$ [12-15] and the $4^{+}$state at $13.3 \mathrm{MeV}$ [16] are considered as the members of the "Hoyle band" $[11,17,18]$. More recently, the $0_{3}^{+}$ state at $10.3 \mathrm{MeV}[13,19]$ is suggested as the "breathing mode" of the Hoyle state [18,20-24]. The possible formation of the $3 \alpha$ linear chain $\left(0_{4}^{+}\right.$state $)$has also been discussed $[18,23]$.

These ideas have been naturally extended to the condensates of many $\alpha$ particles. The candidates of the $4 \alpha$ condensate in ${ }^{16} \mathrm{O}$ are under the intensive discussions [25-32]. However, the theoretical and experimental information for the $5 \alpha, 6 \alpha$, $\ldots$ condensates $[11,33-35]$ is rather scarce. The clustering of the non- $\alpha$ nuclei is another possible extension of these ideas. The Hoyle-analog states with a nucleon hole or particle are discussed for ${ }^{11} \mathrm{~B}[36-39]$ and ${ }^{13} \mathrm{C}[40,41]$. The $3 \alpha$ linear chains accompanied by the valence neutrons are expected in neutron-rich $\mathrm{C}$ isotopes [42-53]. A research interest has now been extended to the higher excited cluster states composing multiclusters and nucleons.

However, with the increase in number of the constituent clusters or nucleons, the description of the cluster states becomes difficult. As in the implementation of the generator coordinate method (GCM) [54,55], it is easy to imagine that the number of basis wave functions required for the description of the cluster states increases very quickly as the number of

*masaaki@nucl.sci.hokudai.ac.jp constituent particles increases or the system becomes dilute. As a result, much computational power is demanded and the practical calculation becomes difficult. This may be one of the reason why the condensates of many $\alpha$ particles are rarely studied based on the microscopic models. Therefore, a new method which efficiently generates the basis wave functions is highly desirable and indispensable. For this purpose, several methods have been developed such as the stochastic sampling of the basis wave functions [56-58] and the imaginary-time development method [59].

In this study, we propose an alternative method which makes use of the equation of motion (EOM) of the Gaussian wave packets. The basis wave functions are generated by the real-time evolution of the system governed by the EOM, and they are superposed to diagonalize the Hamiltonian. The beauty of our method is that it makes use of the real time as the generator coordinate. As a benchmark of the methodology, we applied it to the $3 \alpha$ system $\left({ }^{12} \mathrm{C}\right)$. It is shown that the proposed method works efficiently and yields the results consistent with or better than the other cluster models. Furthermore, based on the isoscalar (IS) monopole and dipole transition strengths, we briefly discuss the structure of the excited $0^{+}$and $1^{-}$ states.

This paper is organized as follows: Section II explains the framework of the proposed method, which we call the real-time evolution method (REM). Our benchmark results are presented and discussed in Sec. III. The conclusion is drawn in Sec. IV.

\section{THEORETICAL FRAMEWORK}

Here, we explain the framework of the REM. For simplicity, we assume its application to the $\alpha$ cluster wave functions ( $4 N$ nuclei). However, it is noted that the method is also applicable to more general cases such as non- $\alpha$ cluster wave functions, antisymmetrized molecular dynamics (AMD) and fermionic molecular dynamics (FMD) wave functions. 


\section{A. Hamiltonian and generator coordinate method wave function}

The Hamiltonian for the $N \alpha$ systems composed of $4 N$ nucleons is given as

$$
\hat{H}=\sum_{i=1}^{4 N} \hat{t}_{i}+\sum_{i<j}^{4 N} \hat{v}_{N}\left(r_{i j}\right)+\sum_{i<j}^{4 N} \hat{v}_{C}\left(r_{i j}\right)-\hat{t}_{c m},
$$

where $\hat{t}_{i}$ and $\hat{t}_{c m}$ respectively denote the kinetic energies of the nucleons and the center of mass. The $\hat{v}_{N}$ and $\hat{v}_{C}$ denote the effective nucleon-nucleon interaction and Coulomb interactions, respectively. The parameter set of $\hat{v}_{N}$ is explained later.

As for the intrinsic wave function of $N \alpha$ system, we employ the Brink-Bloch wave function [60] which is composed of $\alpha$ clusters having $(0 s)^{4}$ configurations,

$$
\begin{aligned}
\Phi\left(\boldsymbol{Z}_{1}, \ldots, \boldsymbol{Z}_{N}\right) & =\mathcal{A}\left\{\Phi_{\alpha}\left(\boldsymbol{Z}_{1}\right) \cdots \Phi_{\alpha}\left(\boldsymbol{Z}_{N}\right)\right\} \\
\Phi_{\alpha}(\boldsymbol{Z}) & =\mathcal{A}\left\{\phi\left(\boldsymbol{r}_{1}, \boldsymbol{Z}\right) \chi_{p \uparrow} \cdots \phi\left(\boldsymbol{r}_{4}, \boldsymbol{Z}\right) \chi_{n \downarrow}\right\} \\
\phi(\boldsymbol{r}, \boldsymbol{Z}) & =\left(\frac{2 v}{\pi}\right)^{3 / 4} \exp \left\{-v\left(\boldsymbol{r}-\frac{\boldsymbol{Z}}{\sqrt{v}}\right)^{2}+\frac{1}{2} Z^{2}\right\}
\end{aligned}
$$

where $\Phi_{\alpha}(\boldsymbol{Z})$ denotes the wave packet describing the $\alpha$ cluster located at $\boldsymbol{Z}$. The set of three-dimensional vectors $\boldsymbol{Z}_{1}, \ldots, \boldsymbol{Z}_{N}$ is complex numbered and describes the $\alpha$-cluster positions in the phase space. The size parameter $v$ of $\alpha$ particle is so chosen to reproduce the observed radius of ${ }^{4} \mathrm{He}(v=0.275)$ which is same with the preceding works [3,18,23,24,61].

Like other cluster models, we superpose the intrinsic wave function having different configurations (different sets of the complex vectors $\left.\boldsymbol{Z}_{1}, \ldots, \boldsymbol{Z}_{N}\right)$ after the parity and the angularmomentum projection (GCM). The most general form of the GCM wave function may be written as

$$
\begin{aligned}
\Psi_{M}^{J \pi}= & \sum_{K} \int d^{3} Z_{1} \cdots d^{3} Z_{N} \\
& \times f_{K}\left(Z_{1}, \ldots, Z_{N}\right) \hat{P}_{M K}^{J \pi} \Phi\left(Z_{1}, \ldots, Z_{N}\right),
\end{aligned}
$$

where $\hat{P}_{M K}^{J \pi}$ is the parity and the angular-momentum projector. The amplitude of the superposition $f_{K}\left(\boldsymbol{Z}_{1}, \ldots, \boldsymbol{Z}_{N}\right)$ must be determined in some ways. As the original Tohsaki-HoriuchiSchuck-Röpke (THSR) wave function ( $J=M=K=0$ ) [9] asserts that the amplitude can be written as

$$
f_{0}\left(\boldsymbol{R}_{1}, \ldots, \boldsymbol{R}_{N}\right)=\prod_{i=1}^{N} \exp \left\{-\frac{1}{2 \beta^{2}} R_{i}^{2}\right\}
$$

where the vectors $Z_{1}, \ldots, Z_{N}$ are reduced to the real-valued vectors $\boldsymbol{R}_{1}, \ldots, \boldsymbol{R}_{N}$, and the parameter $\beta$ controls the size of the $\alpha$-particle condensate. It is known that this THSR ansatz works surprisingly well for the ground and excited $0^{+}$states of ${ }^{12} \mathrm{C}[9-11,61]$.

In other ordinary cluster models, Eq. (5) is often discretized and approximated by a sum of the finite number of the basis wave functions,

$$
\Psi_{M}^{J \pi}=\sum_{p=1}^{p_{\max }} \sum_{K=-J}^{J} f_{p K} \hat{P}_{M K}^{J \pi} \Phi\left(\boldsymbol{Z}_{1}^{(p)}, \ldots, \boldsymbol{Z}_{N}^{(p)}\right),
$$

and the amplitude $f_{p K}$ is calculated by the Griffin-HillWheeler equation $[54,55]$. Here, $\boldsymbol{Z}_{1}^{(p)}, \ldots, \boldsymbol{Z}_{N}^{(p)}$ denotes the $p$ th set of the vectors $\boldsymbol{Z}_{1}, \ldots, \boldsymbol{Z}_{N}$ and the number of the superposed basis wave function is equal to $p_{\max }$. If $p_{\max }$ is sufficiently large and the set of the vectors $\boldsymbol{Z}_{1}^{(p)}, \ldots, \boldsymbol{Z}_{N}^{(p)}$ covers various configurations of $\alpha$ clusters, Eq. (7) will be a good approximation, but the increase of $p_{\max }$ requires much computational cost. It is easy to imagine that the number of basis wave functions, $p_{\max }$, required for a reasonable description of $N \alpha$ systems will be greatly increased, when the number of $\alpha$ particles is increased. This is one of the reasons why the condensates of many $\alpha$ particles are rarely studied with the microscopic models.

Therefore, if one employs the approximation given by Eq. (7), it is essentially important to find a way which efficiently generates the set of the vectors $\boldsymbol{Z}_{1}^{(p)}, \ldots, \boldsymbol{Z}_{N}^{(p)}$ to reduce the computational cost. For this purpose, several methods such as the stochastic method [56-58] and the imaginary time evolution methods [59] have been proposed, and in this study, we introduce a new method which uses the real-time evolution of the $\alpha$-particle wave packets.

\section{B. Real-time evolution method}

In the present study, the EOM of the $\alpha$-particle wave packets is used to generate the sets of the vectors $\boldsymbol{Z}_{1}^{(p)}, \ldots, \boldsymbol{Z}_{N}^{(p)}$. By applying the time-dependent variational principle to the intrinsic wave function given by Eq. (2),

$$
\delta \int d t \frac{\left\langle\Phi\left(\boldsymbol{Z}_{1}, \ldots, \boldsymbol{Z}_{N}\right)|i \hbar d / d t-\hat{H}| \Phi\left(\boldsymbol{Z}_{1}, \ldots, \boldsymbol{Z}_{N}\right)\right\rangle}{\left\langle\Phi\left(\boldsymbol{Z}_{1}, \ldots, \boldsymbol{Z}_{N}\right) \mid \Phi\left(\boldsymbol{Z}_{1}, \ldots, \boldsymbol{Z}_{N}\right)\right\rangle}=0
$$

one obtains the EOM for the $\alpha$ particle centroids $\boldsymbol{Z}_{1}, \ldots, \boldsymbol{Z}_{N}$,

$$
\begin{aligned}
i \hbar \sum_{j=1}^{N} \sum_{\sigma=x, y, z} C_{i \rho j \sigma} \frac{d Z_{j \sigma}}{d t}=\frac{\partial \mathcal{H}_{\text {int }}}{\partial Z_{i \rho}^{*}} \\
\mathcal{H}_{\text {int }} \equiv \frac{\left\langle\Phi\left(\boldsymbol{Z}_{1}, \ldots, \boldsymbol{Z}_{N}\right)|\hat{H}| \Phi\left(\boldsymbol{Z}_{1}, \ldots, \boldsymbol{Z}_{N}\right)\right\rangle}{\left\langle\Phi\left(\boldsymbol{Z}_{1}, \ldots, \boldsymbol{Z}_{N}\right) \mid \Phi\left(\boldsymbol{Z}_{1}, \ldots, \boldsymbol{Z}_{N}\right)\right\rangle}, \\
C_{i \rho j \sigma} \equiv \frac{\partial^{2} \ln \left\langle\Phi\left(\boldsymbol{Z}_{1}, \ldots, \boldsymbol{Z}_{N}\right) \mid \Phi\left(\boldsymbol{Z}_{1}, \ldots, \boldsymbol{Z}_{N}\right)\right\rangle}{\partial Z_{i \rho}^{*} \partial Z_{j \sigma}} .
\end{aligned}
$$

Note that $\boldsymbol{Z}_{1}, \ldots, \boldsymbol{Z}_{A}$ in the bra vector are conjugated in Eqs. (10) and (11). Therefore, $\mathcal{H}_{\text {int }}$ and $C_{i \rho j \sigma}$ are the functions of $\boldsymbol{Z}_{1}, \ldots, \boldsymbol{Z}_{A}$ and $\boldsymbol{Z}_{1}^{*}, \ldots, \boldsymbol{Z}_{A}^{*}$.

Starting from an arbitrary initial wave function at $t=0$, we solve the time evolution of $\boldsymbol{Z}_{1}, \ldots, \boldsymbol{Z}_{N}$. As a result, the EOS yields the set of the vectors $\boldsymbol{Z}_{1}(t), \ldots, \boldsymbol{Z}_{N}(t)$ as a function of time $t$, which defines the wave function $\Phi\left(Z_{1}(t), \ldots, Z_{N}(t)\right)$ at each time. Despite of its classical form, this EOM still holds the information of the quantum system. For example, it was shown that the nuclear phase shift of the $\alpha-\alpha$ scattering can be obtained from the classical trajectory of the wave-packet centroids [62]. In addition to this, it was shown that the ensemble of the wave functions $\Phi\left(Z_{1}(t), \ldots, Z_{N}(t)\right)$ possess the following good properties [63-65] if the nucleon-nucleon collisions and nucleon emission processes are properly treated: 
(1) The ensemble of the time-dependent wave functions $\Phi\left(\boldsymbol{Z}_{1}(t), \ldots, \boldsymbol{Z}_{N}(t)\right)$ has an ergodic nature.

(2) It follows the quantum statistics.

Indeed, on the basis of these EOM properties, the nuclear liquid-gas phase transition during the heavy-ion collisions and the caloric curve for the finite nuclei have been studied [66-69]. Therefore, we expect that, if time is evolved long enough, the ensemble of the wave functions $\Phi\left(\boldsymbol{Z}_{1}(t), \ldots, \boldsymbol{Z}_{N}(t)\right)$ spans a good model space for $N \alpha$ systems. In other words, we expect that the bound and resonant states of $N \alpha$ systems are reasonably described by the superposition of the basis wave functions as follows:

$$
\begin{aligned}
\Psi_{M}^{J \pi}(T)= & \int_{0}^{T} d t \sum_{K=-J}^{J} \hat{P}_{M K}^{J \pi}\left\{f_{K}(t) \Phi\left(\boldsymbol{Z}_{1}(t), \ldots, \boldsymbol{Z}_{N}(t)\right)\right. \\
& \left.+g_{K}(t) \Phi\left(\boldsymbol{Z}_{1}^{*}(t), \ldots, \boldsymbol{Z}_{N}^{*}(t)\right)\right\} .
\end{aligned}
$$

Here, the complex-conjugated basis wave functions are also superposed to properly describe the time-even states. The coefficients $f_{K}(t)$ and $g_{K}(t)$ should be determined by the diagonalization of the Hamiltonian. Eq. (12) can be regarded as the GCM wave function which employs the real-time $t$ as the generator coordinate.

\section{Numerical calculation}

In this study, the REM calculation is performed for the $3 \alpha$ cluster system $\left({ }^{12} \mathrm{C}\right)$. For the sake of the comparison, we used the Volkoff No. 2 effective nucleon-nucleon interaction [70] with a slight modification [2,3], which is common to the other studies using resonating group method (RGM) $[2,3]$ and Tohsaki-Horiuchi-Schuck-Röpke (THSR) wave function $[18,23,61]$. The numerical calculation was performed in the following steps.

(1) In the first step, we randomly generate the $3 \alpha$ cluster wave function and calculate the imaginary-time evolution of the system,

$$
i \hbar \frac{d \boldsymbol{Z}_{i}}{d \tau}=\mu \frac{\partial \mathcal{H}_{\text {int }}}{\partial \boldsymbol{Z}_{i}^{*}},
$$

where $\mu$ is an arbitrary negative number. Equation (13) decreases the intrinsic energy $\mathcal{H}_{\text {int }}$, as the imaginary time $\tau$ is evolved. The imaginary-time evolution is continued until the intrinsic excitation energy,

$$
E_{\text {int }}^{*}=\mathcal{H}_{\text {int }}-\mathcal{H}_{\text {int }}^{\min },
$$

equals a certain value. Here, $\mathcal{H}_{\text {int }}^{\min }$ is the minimum intrinsic energy obtained by the very long imaginarytime evolution, which is $-74.5 \mathrm{MeV}$ in the present Hamiltonian. In the practical calculation, we tested several values of $E_{\text {int }}^{*}(10,20,25$, and $30 \mathrm{MeV})$ and found that $E_{\mathrm{int}}^{*}=25 \mathrm{MeV}$ results in the best convergence.

(2) In the second step, we calculate the real-time evolution [Eq. (9)] starting from the initial wave function obtained in the first step. For the numerical calculation, the time is discretized with an interval of $\Delta t=0.02$

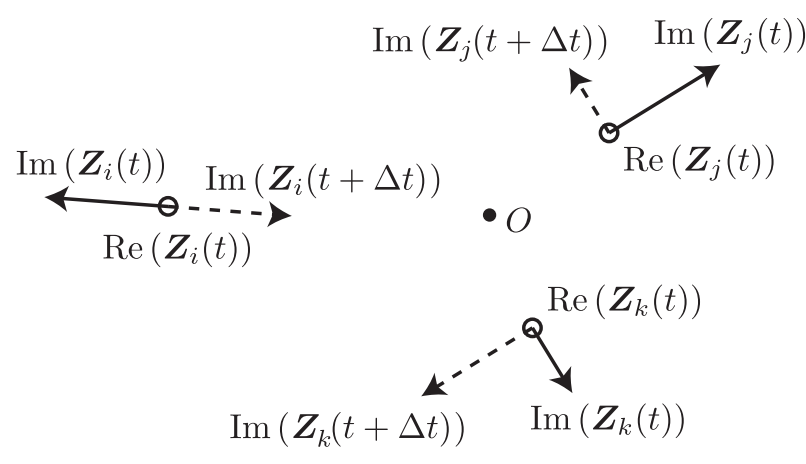

FIG. 1. The rebound of $\alpha$ clusters described by Eqs. (17)-(19). Open circles represent the real part of $\boldsymbol{Z}$, and the solid (dotted) arrows represent the imaginary part of $\boldsymbol{Z}$ before (after) the rebound. The rebound does not change the real parts of $\boldsymbol{Z}_{i}, \boldsymbol{Z}_{j}$, and $\boldsymbol{Z}_{k}$ (positions of $\alpha$ clusters), but changes the imaginary parts (momenta). It reverts the momentum of $\boldsymbol{Z}_{i}$ and the center-of-mass momentum between $\boldsymbol{Z}_{j}$ and $\boldsymbol{Z}_{k}$ but conserves the relative momentum between $\boldsymbol{Z}_{j}$ and $\boldsymbol{Z}_{k}$.

$\mathrm{fm} / c$,

$$
t_{p}=(p-1) \Delta t, \quad p=1,2, \ldots, p_{\max },
$$

and the maximal propagation time is $T_{\max }=\left(p_{\max }-\right.$ 1) $\Delta t=6000 \mathrm{fm} / c\left(p_{\max }=300000\right)$. Note that the intrinsic energy $\mathcal{H}_{\text {int }}$, and hence $E_{\text {int }}^{*}$, is conserved by the EOM. As a result, the time-evolution calculation yields a set of the Brink-Bloch wave functions $\Phi\left(\boldsymbol{Z}_{1}\left(t_{p}\right), \ldots, \boldsymbol{Z}_{N}\left(t_{p}\right)\right), \quad p=1, \ldots, p_{\max }$ having the same $E_{\mathrm{int}}^{*}$. And it is used as the basis wave function of the GCM calculation in the next step.

If $E_{\text {int }}^{*}$ is large enough, $\alpha$ clusters occasionally escape out to infinite distance during the time evolution. This yields basis wave functions having unphysically large radii, which are useless for the description of the bound or resonant states. To avoid this problem, we impose an additional condition on the calculation. When the condition

$$
\max _{i} \operatorname{Re}\left(\left|Z_{i}(t)\right| / \sqrt{v}\right)>R_{\max }
$$

is satisfied, i.e., if any of $\alpha$ clusters is distant more than $R_{\max }$, we interchange their momentum by hand as follows:

$$
\begin{aligned}
& \boldsymbol{Z}_{i}(t+\Delta t)=\operatorname{Re}\left(\boldsymbol{Z}_{i}(t)\right)-i \operatorname{Im}\left(\boldsymbol{Z}_{i}(t)\right), \\
& \boldsymbol{Z}_{j}(t+\Delta t)=\operatorname{Re}\left(\boldsymbol{Z}_{j}(t)\right)-i \operatorname{Im}\left(\boldsymbol{Z}_{k}(t)\right), \\
& \boldsymbol{Z}_{k}(t+\Delta t)=\operatorname{Re}\left(\boldsymbol{Z}_{k}(t)\right)-i \operatorname{Im}\left(\boldsymbol{Z}_{j}(t)\right),
\end{aligned}
$$

where we assume that $\left|\boldsymbol{Z}_{i}(t)\right|>R_{\max }$. It is noted that the real part of $\boldsymbol{Z}(t)$ corresponds to the coordinate of the $\alpha$ cluster, while the imaginary part corresponds to the momentum. As a result, $\alpha$ clusters rebound as illustrated in Fig. 1. In the present calculation, the maximum distance is chosen as $R_{\max }=10.0 \mathrm{fm}$.

(3) The thus-obtained basis wave functions are superposed by using the real-time $t$ as a generator coordinate. In the following we call this step the GCM calculation. 
Since time is discretized, Eq. (12) should read

$$
\Psi_{M}^{J \pi}=\sum_{p=1}^{p_{\max }} \sum_{K=-J}^{J} \hat{P}_{M K}^{J \pi}\left\{f_{K}\left(t_{p}\right) \Phi\left(t_{p}\right)+g_{K}\left(t_{p}\right) \Phi^{*}\left(t_{p}\right)\right\},
$$

where the basis wave function $\Phi\left(\boldsymbol{Z}_{1}\left(t_{p}\right), \ldots, \boldsymbol{Z}_{N}\left(t_{p}\right)\right)$ is abbreviated as $\Phi\left(t_{p}\right)$. The set of the coefficient $f_{K}\left(t_{p}\right), g_{K}\left(t_{p}\right)$ and the eigenenergy are determined by solving the Griffin-Hill-Wheeler equation to diagonalize the Hamiltonian.

In the practical calculation, a set of the basis wave functions $\Phi\left(t_{p}\right)$ obtained by the time evolution is severely redundant. This makes it difficult to solve the Griffin-Hill-Wheeler equation accurately. To avoid this problem, we remove the basis wave functions which have large overlap with others. When a basis wave function $\Phi\left(t_{p}\right)$ satisfies the following condition,

$$
\max _{t<t_{p}} \frac{\left|\left\langle\Phi(t) \mid \Phi\left(t_{p}\right)\right\rangle\right|^{2}}{\langle\Phi(t) \mid \Phi(t)\rangle\left\langle\Phi\left(t_{p}\right) \mid \Phi\left(t_{p}\right)\right\rangle}>O_{\max },
$$

it is removed from the ensemble. Namely, we do not use the basis wave functions which have the overlap with the previous wave functions larger than $O_{\max }$. In the present calculation $O_{\max }$ is chosen as 0.75 . The typical number of basis wave functions selected by Eq. (21) will be discussed in the next section.

(4) As discussed later, the GCM calculation has difficulty describing highly excited broad resonances because of contamination of the nonresonant wave functions. To overcome this problem, we apply the $r^{2}$-constraint method proposed by Funaki et al. [71]. Following this method, we first diagonalize the radius operator,

$$
\begin{gathered}
\sum_{K^{\prime} q}\left\langle\hat{P}_{M K}^{J \pi} \Phi\left(t_{p}\right)\left|\hat{r}^{2}-r_{a}^{2}\right| \hat{P}_{M K^{\prime}}^{J \pi} \Phi\left(t_{q}\right)\right\rangle e_{K^{\prime} q a}=0 \\
\hat{r}^{2}=\sum_{i=1}^{4 N}\left(\boldsymbol{r}_{i}-\boldsymbol{r}_{c m}\right)^{2} /(4 N)
\end{gathered}
$$

which defines a new set of the basis wave functions,

$$
\widetilde{\Phi}_{M a}^{J \pi}=\sum_{K p} e_{K p a} P_{M K}^{J \pi} \Phi\left(t_{p}\right),
$$

corresponding to the eigenvalue $r_{a}^{2}$. Superposing these new basis, we construct the $r^{2}$-constrained GCM wave function,

$$
\Psi_{M}^{J \pi}=\sum_{a\left(r_{a}^{2}<r_{\text {cut }}^{2}\right)}^{\prime}\left\{\widetilde{f}_{a} \widetilde{\Phi}_{M a}^{J \pi}+\widetilde{g}_{a} \widetilde{\Phi}_{M a}^{J \pi *}\right\} .
$$

Here $\sum^{\prime}$ denotes the conditional summation running over all $a$ which satisfy the condition $r_{a}^{2}<r_{\text {cut }}^{2}$. Namely, the basis wave functions which have too-large eigenvalues of the radius operator are excluded. The coefficients $\widetilde{f}_{a}, \widetilde{g}_{a}$ and the eigenenergies are determined by solving the Griffin-Hill-Wheeler equation. It has been shown that this method effectively separates the resonant states from the nonresonant states. In the present calculation, the cutoff radius $r_{\text {cut }}^{2}$ is varied

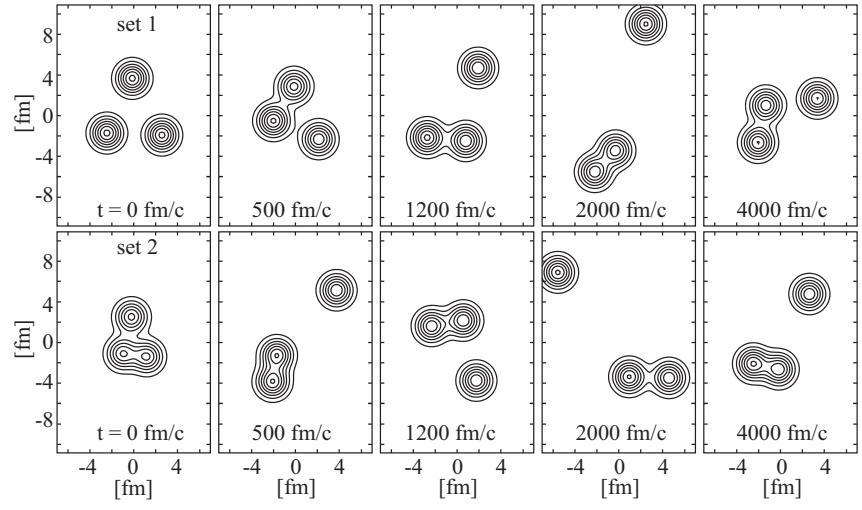

FIG. 2. Intrinsic density snapshots at the propagation time $t=$ $0,500,1200,2000$, and $4000 \mathrm{fm} / c$. Upper (lower) panels show the ensemble of the wave functions obtained by the EOM starting from the wave function at $t=0 \mathrm{fm} / c$.

ranging from $(5.0 \mathrm{fm})^{2}$ to $(7.0 \mathrm{fm})^{2}$ to check the convergence.

\section{NUMERICAL RESULTS}

In this section, we discuss our benchmark results obtained with the proposed method (REM) and compare the results obtained with other models to check the validity and efficiency of the REM. The detailed discussion on the structures of cluster states in ${ }^{12} \mathrm{C}$ will be made in our forthcoming work.

\section{A. Real-time evolution}

As explained in the previous section, the REM relies on the ergodic nature of the EOM. Therefore, if the time is propagated long enough, the results should converge and should not depend on the initial wave functions. To check these points, we tested two different initial wave functions which were generated as follows: First, we set the initial position of the Gaussian wave packets by random numbers. Then, we performed the imaginary-time evolution to change the intrinsic energy $E_{\text {int }}^{*}$ so that it equals $25 \mathrm{MeV}$. Thus, two initial wave functions were generated independently and randomly without any additional input. From these two initial wave functions, we calculated the EOM to yield two independent ensembles of wave functions which are denoted by sets 1 and 2 in the following.

To illustrate how the $3 \alpha$ system is evolved by the EOM, Fig. 2 shows the several wave functions of sets 1 and 2 at particular times. At time $t=0 \mathrm{fm} / c$, the wave function of set 1 has a larger radius but smaller momenta between the $\alpha$ particles compared with set 2 . In both ensembles, disregarding the different initial condition, the $\alpha$ clusters are distributed in various ways: they are close to each other at some time and far distant at another time. Actually, the system repeats a spatial expansion and contraction as time evolves, which can be confirmed from the radius of the system as a function of time as shown in Fig. 3(a). Note that the unphysical change of the expansion velocity at the maximum radius around $9 \mathrm{fm}$ is because of the artificial rebound of the $\alpha$ clusters described 


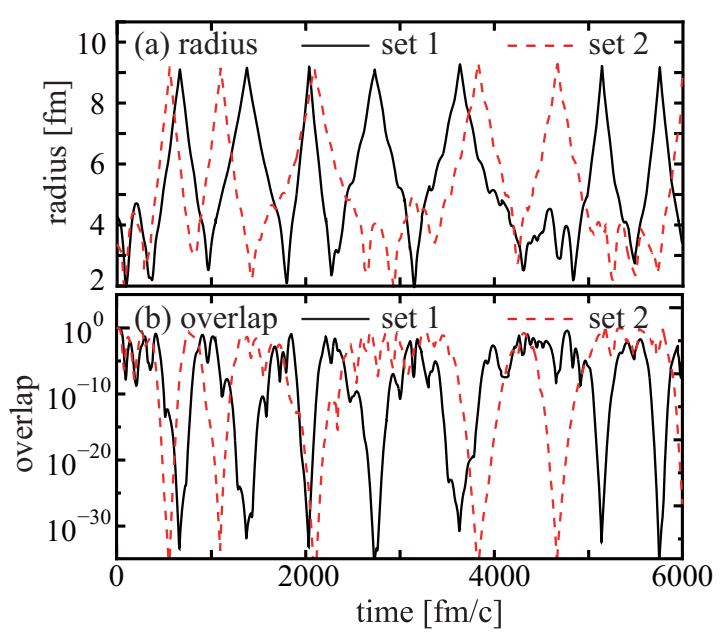

FIG. 3. (a) Radius of the intrinsic wave function as function of time. (b) Overlap between the wave functions at $t=0$ and $t$ after the projection to the $J^{\pi}=0^{+}$.

by Eqs. (17)-(19). Figure 3(b) shows the squared overlap between the wave function $\Phi(t)$ and initial wave function $\Phi(0)$ after the projection to $J^{\pi}=0^{+}$state, which is defined as

$$
\mathcal{O}(t)=\frac{\left|\left\langle\hat{P}^{0^{+}} \Phi(0) \mid \hat{P}^{0^{+}} \Phi(t)\right\rangle\right|^{2}}{\left\langle\hat{P}^{0^{+}} \Phi(0) \mid \hat{P}^{0^{+}} \Phi(0)\right\rangle\left\langle\hat{P}^{0^{+}} \Phi(t) \mid \hat{P}^{0^{+}} \Phi(t)\right\rangle} .
$$

We see that the overlap is rather small, and hence the wave function is almost orthogonal to the initial wave function for most of the time. Thus, the EOM generates various $\alpha$-cluster configurations automatically.

The thus-generated wave functions are selected by the overlap condition represented by Eq. (21). The number of the selected wave functions as function of $T_{\max }$ is shown in Fig. 4. It can be seen that the number is greatly reduced after the selection, because wave functions are heavily redundant. At $T_{\max }=6000 \mathrm{fm} / c$, approximately 250 wave functions are selected out of 300000 in both sets 1 and 2 . In the next section, these selected basis wave functions are superposed to describe the $3 \alpha$ system. It can also be seen that the number is almost saturated at $T_{\max }=5000 \sim 6000 \mathrm{fm} / c$. This indicates

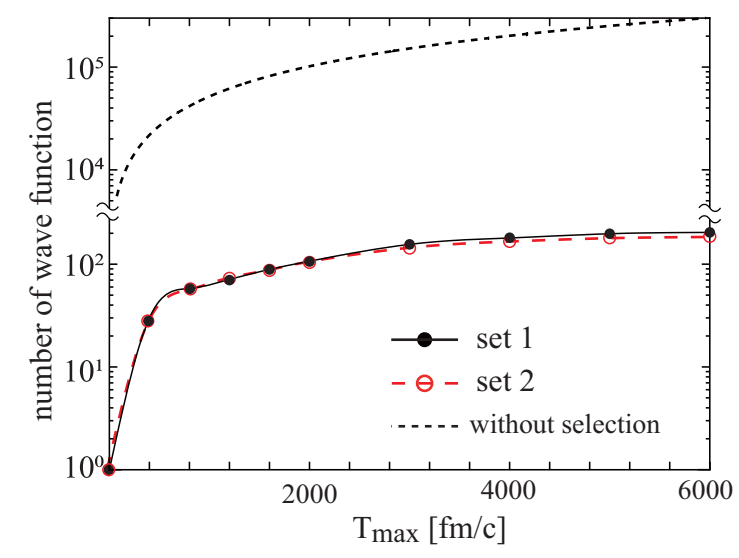

FIG. 4. The number of basis wave functions selected by the overlap condition (21). The black dotted line shows the number of the basis wave functions without the selection.

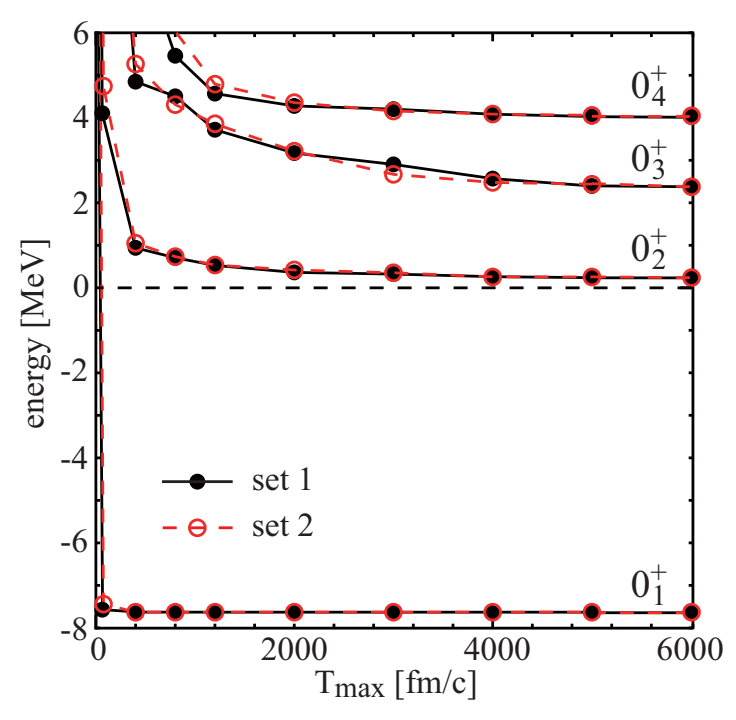

FIG. 5. The eigenenergies of the $0^{+}$states measured from the $3 \alpha$ threshold as a function of the propagation time $T$ obtained by the REM without the $r^{2}$ constraint. Open and filled symbols show the results obtained by using the different initial wave functions at $t=0 \mathrm{fm} / c$.

that almost all possible quantum states are generated after the long-time propagation owing to the ergodic nature of the EOM.

\section{B. Convergence without and with $r^{2}$ constraint}

We first discuss the GCM results obtained without the $r^{2}$ constraint. Figure 5 shows the energies of the $0^{+}$states as a function of the maximum propagation time $T_{\max }$. We see that the energies of the $0^{+}$states converge and are independent of the initial wave functions, if the propagation time is long enough. In particular, the energy of the ground state converges very quickly, despite the rather high intrinsic excitation energy $\left(E_{\text {int }}^{*}=25 \mathrm{MeV}\right)$ of the basis wave functions generated by the EOM. We also found that the quick convergence is common to another bound state $\left(2_{1}^{+}\right.$state).

On the other hand, the energy convergence of the excited $0^{+}$states is not as fast as that of the ground state. In particular, it is interesting to note that the convergence of the $0_{3}^{+}$state looks much slower than others. This is related to the fact that the $0_{3}^{+}$state is a very broad resonance $[13,19]$. Furthermore, if we observe the figure carefully, we find that the energies of these unbound states still go down even at large $T_{\max }$. This is because of the contamination of the nonresonant wave functions to these excited $0^{+}$states, which can be seen more clearly in the $T_{\max }$ dependence of the radius shown in Fig. 6. Here again we see that the convergence of the ground state is surprisingly fast as compared with other unbound states. In this figure, we clearly observe that the radii of the unbound states continuously increase, showing the contamination of the nonresonant wave functions.

To avoid the contamination of the nonresonant wave functions, we applied the $r^{2}$ constraint [71]. This prescription excludes the basis wave functions with huge radii and makes it possible to obtain the approximate energies and the wave 


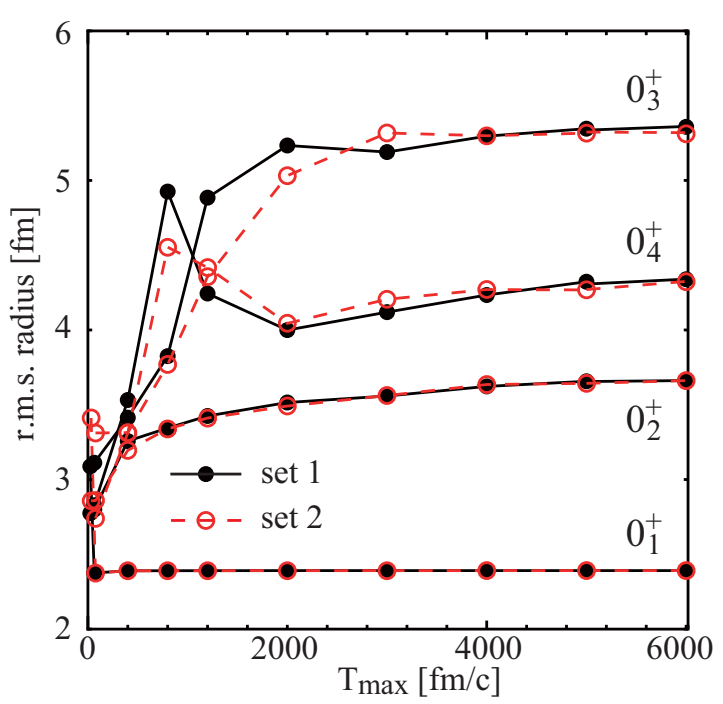

FIG. 6. Same as Fig. 5 but for radii.

functions of the resonant states. Since the $r^{2}$ constraint was already applied to the THSR wave function $[18,18,24]$, it is worthwhile to compare the results of THSR and REM. In the following calculations, the total propagation time $T_{\max }$ is fixed to $6000 \mathrm{fm} / c$.

The top panel of Fig. 7 shows the number of the basis wave functions whose radii are smaller than the cutoff radius $r_{\text {cut }}$ [see Eqs. (22)-(25)]. It is approximately 130 and 220 at $r_{\text {cut }}=$ 5.0 and $7.0 \mathrm{fm}$, respectively. As seen in the middle and bottom panels of Fig. 7, the energies of the $0_{2}^{+}$and $0_{4}^{+}$states are ap- proximately constant in the region of the $r_{\mathrm{cut}} \geqslant 6 \mathrm{fm}$, and the radii increase very slowly as a function of the $r_{\text {cut }}$. This implies that the most of the resonant wave functions in the interaction region is already described by the basis wave functions with $r_{\text {cut }}<6 \mathrm{fm}$, and the choice of the $6 \leqslant r_{\text {cut }} \leqslant 7 \mathrm{fm}$ may give reasonable approximation for the $0_{2}^{+}$and $0_{4}^{+}$states. We also note the results for the $0_{2}^{+}$and $0_{4}^{+}$look almost consistent with the THSR results. On the other hand, we have not obtained the reasonable convergence for the $0_{3}^{+}$state. In particular, the radius continues to increase as function of $r_{\text {cut }}$ not only in the REM calculation but also in the THSR calculation, which implies the contamination of the nonresonant wave functions. This requires more sophisticated method such as the complex scaling for more precise discussion of this state [20-22].

Finally, Fig. 8 shows the electric monopole transition matrix element between the ground and excited $0^{+}$states. Again we see that the Hoyle state is quite stable, while the $0_{3}^{+}$and $0_{4}^{+}$are dependent on $r_{\text {cut }}$. Since the monopole matrix element is very sensitive to the tail of the wave functions, this behavior also indicates the non-negligible contamination of the continuum states with large radii.

\section{Excitation spectrum of ${ }^{12} \mathrm{C}$}

Here, we discuss the excitation spectrum of ${ }^{12} \mathrm{C}$ and make brief comments on the structure of several states. Figure 9 shows the excitation spectrum of ${ }^{12} \mathrm{C}$ calculated by the REM with the ensemble set 1 and $r_{\text {cut }}=6.4 \mathrm{fm}$ together with the results calculated by the RGM $[2,3]$ and THSR $[18,23]$. Their energies and radii are also listed in Table I. Note that all of these calculations use the same Hamiltonian, and hence they should be consistent to each other, and the deeper binding

TABLE I. Calculated and observed energies measured from $3 \alpha$ threshold in MeV and radii in fm. Experimental data are taken from Refs. [13-16,19,72-76].

\begin{tabular}{|c|c|c|c|c|c|c|c|c|}
\hline \multirow[t]{2}{*}{$J^{\pi}$} & \multicolumn{2}{|c|}{ REM } & \multicolumn{2}{|c|}{ THSR $[11,18,23]$} & \multicolumn{2}{|c|}{ RGM [2,3] } & \multicolumn{2}{|c|}{ EXPT } \\
\hline & E & $\sqrt{\left\langle r^{2}\right\rangle}$ & E & $\sqrt{\left\langle r^{2}\right\rangle}$ & E & $\sqrt{\left\langle r^{2}\right\rangle}$ & E & $\sqrt{\left\langle r^{2}\right\rangle}$ \\
\hline $0_{1}^{+}$ & -7.6 & 2.4 & -7.5 & 2.4 & -7.4 & 2.4 & -7.3 & 2.4 \\
\hline $2_{1}^{+}$ & -5.1 & 2.4 & -4.8 & 2.4 & -4.6 & 2.4 & -2.8 & \\
\hline $4_{2}^{+}$ & 1.0 & 2.3 & 2.2 & 2.3 & 2.0 & 2.3 & 6.8 & \\
\hline $0_{2}^{+}$ & 0.3 & 3.7 & 0.2 & 3.7 & 0.4 & 3.5 & 0.4 & \\
\hline $2_{2}^{+}$ & 1.7 & 3.9 & 1.6 & 3.9 & 2.1 & 4.0 & 2.8 & \\
\hline $4_{1}^{+}$ & 3.8 & 4.5 & 3.7 & 4.5 & & & 6.0 & \\
\hline $0_{3}^{+}$ & 2.8 & 4.6 & 2.7 & 4.7 & & & 1.8 & \\
\hline $2_{3}^{+}$ & 3.9 & 4.6 & 4.0 & 4.5 & & & 3.9 & \\
\hline $4_{3}^{+}$ & 5.4 & 4.8 & 5.6 & 4.7 & & & & \\
\hline $0_{4}^{+}$ & 4.0 & 4.2 & 3.9 & 4.2 & & & & \\
\hline $2_{4}^{+}$ & 4.6 & 3.7 & 4.3 & 4.1 & & & & \\
\hline $4_{4}^{+}$ & 6.6 & 5.0 & 6.8 & 4.7 & & & & \\
\hline $3_{1}^{-}$ & 0.4 & 2.8 & & & 0.8 & 2.8 & 2.4 & \\
\hline $4_{1}^{-}$ & 4.1 & 2.9 & & & & & 6.1 & \\
\hline $5_{1}^{-}$ & 12 & 3.6 & & & & & 15 & \\
\hline $1_{1}^{-}$ & 2.8 & 4.3 & & & 3.4 & 3.4 & 3.6 & \\
\hline $2_{1}^{-}$ & 4.0 & 3.5 & & & & & 4.6 & \\
\hline $3_{2}^{-}$ & 5.4 & 4.5 & & & & & & \\
\hline $4_{2}^{-}$ & 6.4 & 4.7 & & & & & & \\
\hline $5_{2}^{-}$ & 9.3 & 4.5 & & & & & & \\
\hline
\end{tabular}




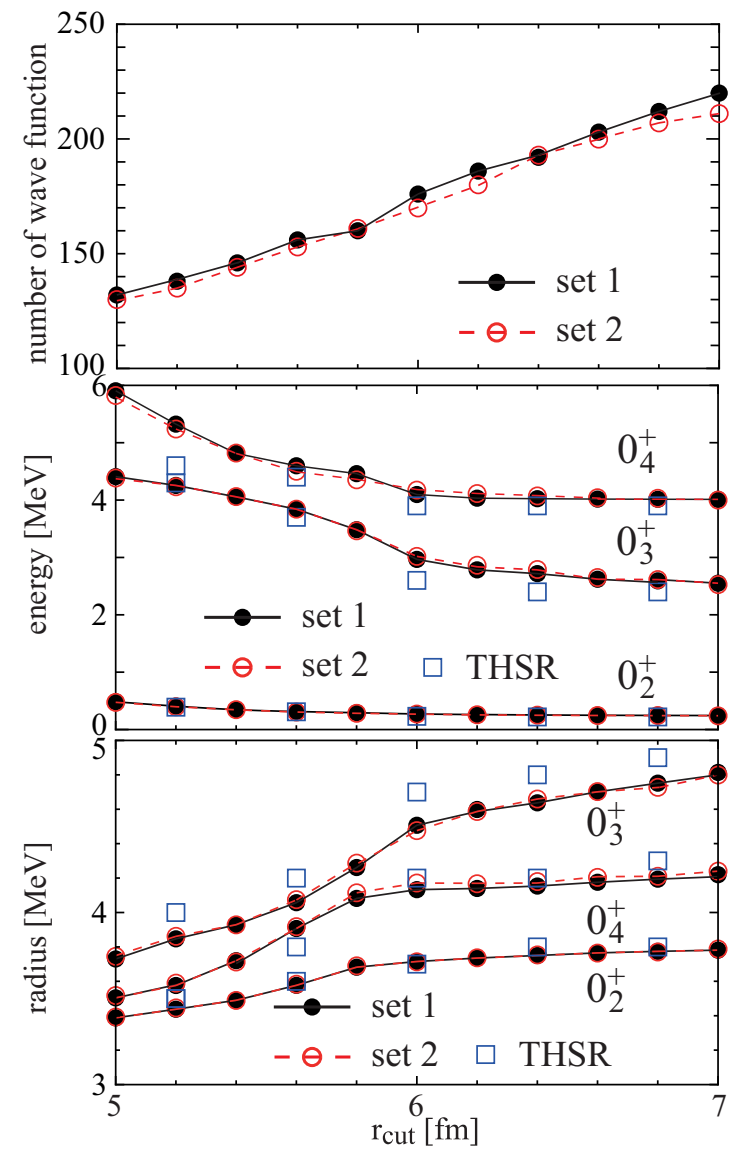

FIG. 7. The number of the basis wave functions (top), the energies (middle) and radius (bottom) of the excited $0^{+}$states as the functions of the cutoff radius $r_{\text {cut }}$. THSR results are taken from Ref. [18].

energy means a better wave function for the bound states. We see that all of the theoretical results are qualitatively consistent with each other. In particular, REM and THSR results agre reasonably for all positive-parity states which include the compact shell-model-like ground band and highly

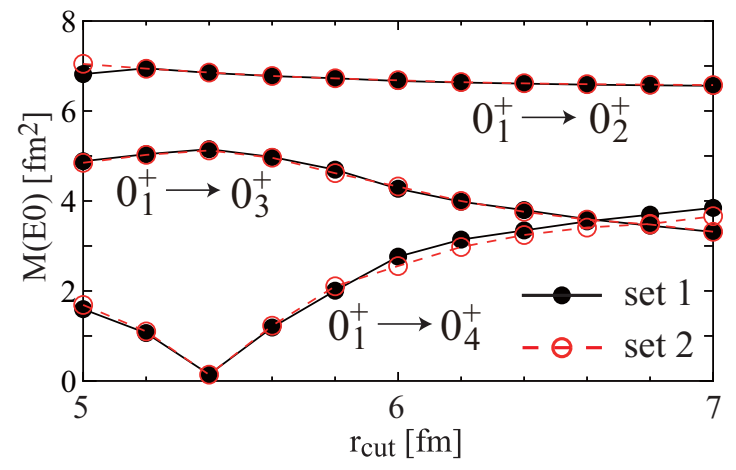

FIG. 8. Electric monopole transition matrix element from the ground state to the excited $0^{+}$states obtained by the $r^{2}$ constraint.

excited cluster states. As for the negative-parity states, REM and RGM reasonably agree for the $1_{1}^{-}$and $3_{1}^{-}$states, and REM additionally produces the $2_{1}^{-}, 3_{2}^{-}$, and $4_{1}^{-}$states, which are also described by AMD [5,8] and FMD [7]. In short, REM can describe all of the states reported by THSR and RGM reasonably. It must be emphasized that not only the $0^{+}$states but all of the states shown in Fig. 9 were obtained from a single ensemble set 1 , which means that the EOM effectively generates the basis wave functions.

Now, we discuss the energies and radii listed in Table I. First, for the bound states $\left(0_{1}^{+}\right.$and $2_{1}^{+}$states), we see that REM yields deeper binding energies than THSR and RGM. This may be due to the limitation of the model space of the THSR and RGM calculations. Namely, the THSR calculation assumes the axially symmetric intrinsic state and RGM calculation limits the relative angular momentum between clusters up to 2, while REM has no such assumptions. Second, the REM and THSR results for the highly excited resonances, in particular the $2_{4}^{+}$and $4_{4}^{+}$states, do not agree perfectly, despite of the use of the same $r^{2}$-constraint method. This shows the difficulty in describing the broad resonances within the bound-state approximation. In particular, since the radius cannot be defined for resonances, we need other measure to discuss the size of these broad resonances.

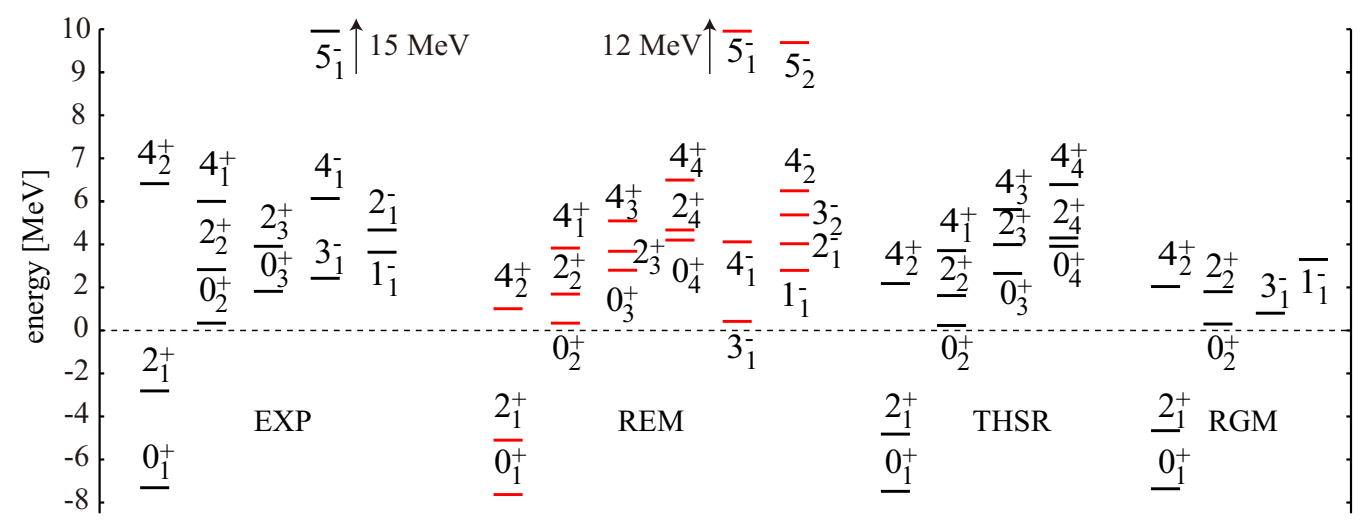

FIG. 9. Excitation energies measured from the $3 \alpha$ threshold. Theoretical results by the REM, THSR [18,23] and RGM [2,3] are compared with the experiments $[13-16,19,72-76]$. The calculated $4_{1}^{+}, 4_{2}^{+}, 5_{1}^{-}$, and $5_{2}^{-}$states are labeled according to the observed counterparts. 
TABLE II. Calculated and observed electric monopole and isoscalar dipole transition matrix elements in the units of $e \mathrm{fm}^{2}$ and $\mathrm{fm}^{3}$.

\begin{tabular}{lcccc}
\hline \hline Transition & REM & THSR [11,18,23] & RGM [2,3] & EXPT [77] \\
\hline $0_{1}^{+} \rightarrow 0_{2}^{+}$ & 6.4 & 6.3 & 6.7 & $5.4 \pm 2$ \\
$0_{1}^{+} \rightarrow 0_{3}^{+}$ & 3.8 & 3.9 & & \\
$0_{1}^{+} \rightarrow 0_{4}^{+}$ & 3.3 & 3.5 & & \\
$0_{2}^{+} \rightarrow 0_{3}^{+}$ & 28 & 34 & & \\
$0_{2}^{+} \rightarrow 0_{4}^{+}$ & 0.7 & 0.5 & & \\
$0_{1}^{+} \rightarrow 1_{1}^{-}$ & 3.7 & & \\
$0_{2}^{+} \rightarrow 1_{1}^{-}$ & 45 & & \\
\hline \hline
\end{tabular}

Finally, we discuss the characteristics of the excited $0^{+}$ and $1^{-}$states referring their electric monopole and isoscalar dipole transition strengths. The transition matrix elements are defined as

$$
\begin{aligned}
& M\left(E 0 ; 0_{m}^{+} \rightarrow 0_{n}^{+}\right)=\left\langle 0_{n}^{+}\left\|\sum_{i=1}^{A} r_{i}^{\prime 2} \frac{1+\tau_{z i}}{2}\right\| 0_{m}^{+}\right\rangle, \\
& M\left(I S 1 ; 0_{m}^{+} \rightarrow 1_{n}^{-}\right)=\left\langle 1_{n}^{-}\left\|\sum_{i=1}^{A} r_{i}^{\prime 3} Y_{1}\left(\hat{r}_{i}^{\prime}\right)\right\| 0_{m}^{+}\right\rangle,
\end{aligned}
$$

where $\boldsymbol{r}_{i}^{\prime}$ denotes the single-particle coordinate measured from the center of mass. The results are summarized in Table II.

Since the monopole transition operator is nothing but the radius operator, the matrix element should be large for the dilute gas-like states [78]. Indeed, it is well known that the Hoyle state has the enhanced monopole transition strength from the ground state because of its dilute gas-like nature. The present calculation yields $6.4 e \mathrm{fm}^{2}$ (1.5 WU), which is consistent with the other cluster models but slightly overestimates the observation. The monopole transition from the ground state to the more dilute $0_{3}^{+}$state is also large and comparable with the Weisskopf unit (WU), but not as large as that of the Hoyle state. The reason of the reduction is that the $0_{3}^{+}$state is dominantly composed of the $4 \hbar \omega$ configurations which cannot be excited by the monopole operator $(2 \hbar \omega$ excitation). However, it must be noted that the transition from the Hoyle state to the $0_{3}^{+}$state is greatly enhanced (6.2 WU). From this result and from the analysis of the wave function, it was concluded that the $0_{3}^{+}$state is a $2 \hbar \omega$ excited state built on the Hoyle state $[18,23]$. In other words, it is the breathing mode of the Hoyle state [24]. This relationship between the ground, Hoyle, and $0_{3}^{+}$states is schematically illustrated in Fig. 10. On the contrary, the monopole transition between the Hoyle state and the $0_{4}^{+}$state is rather weak. This is due to the structural mismatch between these states. In Ref. [23], it was concluded that the $\alpha$ clusters are linearly aligned in the $0_{4}^{+}$ state (linear-chain state), which naturally reduces the overlap with the Hoyle state.

A finding in the present study suggests that not only the $0_{3}^{+}$ state but also the $1_{1}^{-}$state may be an excited state of the Hoyle state. As discussed in Ref. [79], the $1^{-}$states with pronounced clustering should have the strong isoscalar dipole transition strength from the ground state. The present calculation yields

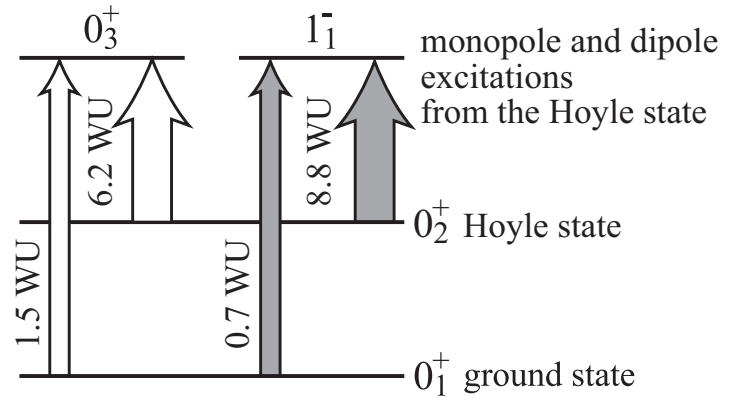

FIG. 10. Excitation modes of the Hoyle state are schematically shown. Arrows show the monopole and dipole transitions.

$M\left(I S 1 ; 0_{1}^{+} \rightarrow 1_{1}^{-}\right)=3.7 \mathrm{fm}^{3}$, which is comparable to the Weisskopf unit $(0.7 \mathrm{WU})$. A similar strength was also obtained by an AMD calculation [80]. Furthermore, note that the isoscalar dipole transition between the Hoyle state and the $1_{1}^{-}$state is extraordinary strong ( $\left.8.8 \mathrm{WU}\right)$. From this result, we are tempted to conclude that the $1_{1}^{-}$state is the $1 \hbar \omega$ (or $3 \hbar \omega$ ) excitation of the Hoyle state. Indeed, the $1_{1}^{-}$state has a huge radius comparable to the $0_{3}^{+}$state. It is also interesting to note that the $1_{1}^{-}$state is energetically very close to the $0_{3}^{+}$ state. This conjecture is also illustrated in Fig. 10.

\section{SUMMARY}

In summary, we developed a theoretical model which makes use of the classical EOM of the Gaussian centroids to generate the ergodic ensemble of the basis wave functions. The generated basis wave functions are superposed to diagonalize the Hamiltonian. Thus, the method named REM can be regarded as a generator coordinate method which employs the real time $t$ as the generator coordinate.

As a benchmark of REM, we applied it to the $3 \alpha$ system $\left({ }^{12} \mathrm{C}\right)$ and found that the results are consistent with or even better than the other cluster models. It was shown that, when the propagation time is long enough, the energies and the radii of the ground and many excited states converge and are independent of the initial condition. As a result, REM successfully described all of the states reported by THSR and RGM. It must be emphasized that all of the states are obtained from a single ensemble of the basis wave function, which indicates that the EOM effectively generates the basis wave functions. However, even if we apply the $r^{2}$ constraint, several excited states were not well converged because of the contamination of the nonresonant wave function. A particular case is the $0_{3}^{+}$state which has broad width and is regarded as the breathing mode of the Hoyle state. A more accurate description of these states requires further development of the method.

Based on the electric monopole and isoscalar dipole transition strengths, the characteristics of the excited $0^{+}$and $1^{-}$ states are discussed. We confirmed that the properties of the Hoyle state and the $0_{3}^{+}$states are consistent with those discussed in the preceding studies. They have dilute structure and the enhanced monopole transition strengths from the ground state. The huge monopole transition strength between the 
Hoyle state and the $0_{3}^{+}$state was also confirmed. In addition to this, we found that the $1_{1}^{-}$state has properties analogous to those of the $0_{3}^{+}$state. Namely, the $1_{1}^{-}$state has dilute structure and the enhanced dipole transition strengths from the ground state. It also has the extraordinary large isoscalar dipole strength from the Hoyle state. From these results, we conjecture that the $1_{1}^{-}$state can be also regarded as an excitation mode of the Hoyle state. Although this conjecture is based on only the transition strengths and the overlaps, a more detailed quantitative discussion based on the reduced width amplitudes, transition form factors, and occupation probabilities will be made in our forthcoming presentations.

\section{ACKNOWLEDGMENTS}

The authors acknowledge fruitful discussions with Dr. Zhou, Dr. Funaki, Dr. Horiuchi, and Dr. Kawabata. One of the authors (M.K.) acknowledges that this work was initiated by the discussion with Dr. Kanada-En'yo and Dr. Yabana. He thanks Dr. Jagjit for careful proofreading and valuable comments. This work was supported by Japan Society for the Promotion of Sciences KAKENHI Grants No. 16K05339 and No. 19K03859, the collaborative research programs 2019 at the Hokkaido University information initiative center, and by the grant for the RCNP joint research project.
[1] E. Uegaki, Y. Abe, S. Okabe, and H. Tanaka, Prog. Theor. Phys. 59, 1031 (1978).

[2] Y. Fujiwara, H. Horiuchi, K. Ikeda, M. Kamimura, K. Kato, Y. Suzuki, and E. Uegaki, Prog. Theor. Phys. Suppl. 68, 29 (1980).

[3] M. Kamimura, Nucl. Phys. A 351, 456 (1981).

[4] P. Descouvemont and D. Baye, Phys. Rev. C 36, 54 (1987).

[5] Y. Kanada-En'yo, Phys. Rev. Lett. 81, 5291 (1998).

[6] P. Descouvemont, C. Daniel, and D. Baye, Phys. Rev. C 67, 044309 (2003).

[7] M. Chernykh, H. Feldmeier, T. Neff, P. von Neumann-Cosel, and A. Richter, Phys. Rev. Lett. 98, 032501 (2007).

[8] Y. Kanada-En'yo, Prog. Theor. Phys. 117, 655 (2007).

[9] A. Tohsaki, H. Horiuchi, P. Schuck, and G. Röpke, Phys. Rev. Lett. 87, 192501 (2001).

[10] T. Yamada, Y. Funaki, H. Horiuchi, G. Röpke, P. Schuck, and A. Tohsaki, in Lecture Notes Physics Cluster Nuclei, edited by C. Beck (Springer, Berlin, Heidelberg, 2012), Vol. 2, Chap. 5, pp. 229-298.

[11] P. Schuck, Y. Funaki, H. Horiuchi, G. Röpke, A. Tohsaki, and T. Yamada, Phys. Scr. 91, 123001 (2016).

[12] M. Freer, H. Fujita, Z. Buthelezi, J. Carter, R. W. Fearick, S. V. Förtsch, R. Neveling, S. M. Perez, P. Papka, F. D. Smit, J. A. Swartz, and I. Usman, Phys. Rev. C 80, 041303(R) (2009).

[13] M. Itoh, H. Akimune, M. Fujiwara, U. Garg, N. Hashimoto, T. Kawabata, K. Kawase, S. Kishi, T. Murakami, K. Nakanishi, Y. Nakatsugawa, B. K. Nayak, S. Okumura, H. Sakaguchi, H. Takeda, S. Terashima, M. Uchida, Y. Yasuda, M. Yosoi, and J. Zenihiro, Phys. Rev. C 84, 054308 (2011).

[14] W. R. Zimmerman, N. E. Destefano, M. Freer, M. Gai, and F. D. Smit, Phys. Rev. C 84, 027304 (2011).

[15] W. R. Zimmerman, M. W. Ahmed, B. Bromberger, S. C. Stave, A. Breskin, V. Dangendorf, T. Delbar, M. Gai, S. S. Henshaw, J. M. Mueller, C. Sun, K. Tittelmeier, H. R. Weller, and Y. K. Wu, Phys. Rev. Lett. 110, 152502 (2013).

[16] M. Freer, S. Almaraz-Calderon, A. Aprahamian, N. I. Ashwood, M. Barr, B. Bucher, P. Copp, M. Couder, N. Curtis, X. Fang, F. Jung, S. Lesher, W. Lu, J. D. Malcolm, A. Roberts, W. P. Tan, C. Wheldon, and V. A. Ziman, Phys. Rev. C 83, 034314 (2011).

[17] M. Freer and H. Fynbo, Prog. Part. Nucl. Phys. 78, 1 (2014).

[18] Y. Funaki, Phys. Rev. C 92, 021302(R) (2015).

[19] M. Itoh, H. Akimune, M. Fujiwara, U. Garg, T. Kawabata, K. Kawase, T. Murakami, K. Nakanishi, Y. Nakatsugawa, H. Sakaguchi, S. Terashima, M. Uchida, Y. Yasuda, M. Yosoi, and J. Zenihiro, J. Phys.: Conf. Ser. 436, 012006 (2013).
[20] C. Kurokawa and K. Kato, Phys. Rev. C 71, 021301(R) (2005).

[21] C. Kurokawa and K. Kato, Nucl. Phys. A 792, 87 (2007).

[22] S.-I. Ohtsubo, Y. Fukushima, M. Kamimura, and E. Hiyama, Prog. Theor. Exp. Phys. 2013, 073 D02 (2013).

[23] Y. Funaki, Phys. Rev. C 94, 024344 (2016).

[24] B. Zhou, A. Tohsaki, H. Horiuchi, and Z. Ren, Phys. Rev. C 94, 044319 (2016).

[25] T. Wakasa, E. Ihara, K. Fujita, Y. Funaki, K. Hatanaka, H. Horiuchi, M. Itoh, J. Kamiya, G. Röpke, H. Sakaguchi, N. Sakamoto, Y. Sakemi, P. Schuck, Y. Shimizu, M. Takashina, S. Terashima, A. Tohsaki, M. Uchida, H. Yoshida, and M. Yosoi, Phys. Lett. B 653, 173 (2007).

[26] Y. Funaki, T. Yamada, H. Horiuchi, G. Röpke, P. Schuck, and A. Tohsaki, Phys. Rev. Lett. 101, 082502 (2008).

[27] N. Curtis, S. Almaraz-Calderon, A. Aprahamian, N. I. Ashwood, M. Barr, B. Bucher, P. Copp, M. Couder, X. Fang, M. Freer, G. Goldring, F. Jung, S. R. Lesher, W. Lu, J. D. Malcolm, A. Roberts, W. P. Tan, C. Wheldon, and V. A. Ziman, Phys. Rev. C 88, 064309 (2013).

[28] M. R. D. Rodrigues, T. Borello-Lewin, H. Miyake, J. L. M. Duarte, C. L. Rodrigues, M. A. Souza, L. B. HorodynskiMatsushigue, G. M. Ukita, F. Cappuzzello, A. Cunsolo, M. Cavallaro, C. Agodi, and A. Foti, Phys. Rev. C 89, 024306 (2014).

[29] R. Bijker and F. Iachello, Phys. Rev. Lett. 112, 152501 (2014).

[30] A. A. Ogloblin, A. N. Danilov, A. S. Demyanova, S. A. Goncharov, and T. L. Belyaeva, Phys. Rev. C 94, 051602(R) (2016).

[31] R. Bijker and F. Iachello, Nucl. Phys. A 957, 154 (2017).

[32] K. C. W. Li, R. Neveling, P. Adsley, P. Papka, F. D. Smit, J. W. Brümmer, C. A. Diget, M. Freer, M. N. Harakeh, T. Kokalova, F. Nemulodi, L. Pellegri, B. Rebeiro, J. A. Swartz, S. Triambak, J. J. van Zyl, and C. Wheldon, Phys. Rev. C 95, 031302(R) (2017).

[33] T. Yamada and P. Schuck, Phys. Rev. C 69, 024309 (2004).

[34] T. Kokalova, N. Itagaki, W. von Oertzen, and C. Wheldon, Phys. Rev. Lett. 96, 192502 (2006).

[35] N. Itagaki, M. Kimura, C. Kurokawa, M. Ito, and W. von Oertzen, Phys. Rev. C 75, 037303 (2007).

[36] P. Descouvemont, Nucl. Phys. A 584, 532 (1995).

[37] Y. Kanada-En'yo, Phys. Rev. C 75, 024302 (2007).

[38] T. Kawabata, H. Akimune, H. Fujita, Y. Fujita, M. Fujiwara, K. Hara, K. Hatanaka, M. Itoh, Y. Kanada-En'yo, S. Kishi, K. Nakanishi, H. Sakaguchi, Y. Shimbara, A. Tamii, S. Terashima, M. Uchida, T. Wakasa, Y. Yasuda, H. Yoshida, and M. Yosoi, Phys. Lett. B 646, 6 (2007). 
[39] T. Yamada and Y. Funaki, Phys. Rev. C 82, 064315 (2010).

[40] T. Yamada, H. Horiuchi, and P. Schuck, Mod. Phys. Lett. A 21, 2373 (2006).

[41] T. Yamada and Y. Funaki, Phys. Rev. C 92, 034326 (2015).

[42] N. Itagaki, S. Okabe, K. Ikeda, and I. Tanihata, Phys. Rev. C 64 , 014301 (2001).

[43] T. Suhara and Y. Kanada-En'yo, Phys. Rev. C 82, 044301 (2010).

[44] M. Freer, J. D. Malcolm, N. L. Achouri, N. I. Ashwood, D. W. Bardayan, S. M. Brown, W. N. Catford, K. A. Chipps, J. Cizewski, N. Curtis, K. L. Jones, T. Munoz-Britton, S. D. Pain, N. Soić, C. Wheldon, G. L. Wilson, and V. A. Ziman, Phys. Rev. C 90, 054324 (2014).

[45] T. Baba, Y. Chiba, and M. Kimura, Phys. Rev. C 90, 064319 (2014).

[46] J.-P. Ebran, E. Khan, T. Nikšić, and D. Vretenar, Phys. Rev. C 90, 054329 (2014).

[47] D. Dell'Aquila, I. Lombardo, L. Acosta, R. Andolina, L. Auditore, G. Cardella, M. B. Chatterjiee, E. De Filippo, L. Francalanza, B. Gnoffo, G. Lanzalone, A. Pagano, E. V. Pagano, M. Papa, S. Pirrone, G. Politi, F. Porto, L. Quattrocchi, F. Rizzo, E. Rosato, P. Russotto, A. Trifirò, M. Trimarchi, G. Verde, and M. Vigilante, Phys. Rev. C 93, 024611 (2016).

[48] A. Fritsch, S. Beceiro-Novo, D. Suzuki, W. Mittig, J. J. Kolata, T. Ahn, D. Bazin, F. D. Becchetti, B. Bucher, Z. Chajecki, X. Fang, M. Febbraro, A. M. Howard, Y. Kanada-En'yo, W. G. Lynch, A. J. Mitchell, M. Ojaruega, A. M. Rogers, A. Shore, T. Suhara, X. D. Tang, R. Torres-Isea, and H. Wang, Phys. Rev. C 93, 014321 (2016).

[49] Z. Y. Tian, Y. L. Ye, Z. H. Li, C. J. Lin, Q. T. Li, Y. C. Ge, J. L. Lou, W. Jiang, J. Li, Z. H. Yang, J. Feng, P. J. Li, J. Chen, Q. Liu, H. L. Zang, B. Yang, Y. Zhang, Z. Q. Chen, Y. Liu, X. H. Sun, J. Ma, H. M. Jia, X. X. Xu, L. Yang, N. R. Ma, and L. J. Sun, Chin. Phys. C 40, 111001 (2016).

[50] T. Baba and M. Kimura, Phys. Rev. C 94, 044303 (2016).

[51] J. Li, Y. L. Ye, Z. H. Li, C. J. Lin, Q. T. Li, Y. C. Ge, J. L. Lou, Z. Y. Tian, W. Jiang, Z. H. Yang, J. Feng, P. J. Li, J. Chen, Q. Liu, H. L. Zang, B. Yang, Y. Zhang, Z. Q. Chen, Y. Liu, X. H. Sun, J. Ma, H. M. Jia, X. X. Xu, L. Yang, N. R. Ma, and L. J. Sun, Phys. Rev. C 95, 021303(R) (2017).

[52] H. Yamaguchi, D. Kahl, S. Hayakawa, Y. Sakaguchi, K. Abe, T. Nakao, T. Suhara, N. Iwasa, A. Kim, D. Kim, S. Cha, M. Kwag, J. Lee, E. Lee, K. Chae, Y. Wakabayashi, N. Imai, N. Kitamura, P. Lee, J. Moon, K. Lee, C. Akers, H. Jung, N. Duy, L. Khiem, and C. Lee, Phys. Lett. B 766, 11 (2017).

[53] T. Baba and M. Kimura, Phys. Rev. C 95, 064318 (2017).

[54] D. L. Hill and J. A. Wheeler, Phys. Rev. 89, 1102 (1953).

[55] J. J. Griffin and J. A. Wheeler, Phys. Rev. 108, 311 (1957).

[56] Y. Suzuki and K. Varga, in Stochastic Variable Approach to Quantum-Mechanical Few-Body Problem (Springer, Berlin, Heidelberg, 1998).
[57] N. Itagaki, A. Kobayakawa, and S. Aoyama, Phys. Rev. C 68, 054302 (2003).

[58] J. Mitroy, S. Bubin, W. Horiuchi, Y. Suzuki, L. Adamowicz, W. Cencek, K. Szalewicz, J. Komasa, D. Blume, and K. Varga, Rev. Mod. Phys. 85, 693 (2013).

[59] Y. Fukuoka, S. Shinohara, Y. Funaki, T. Nakatsukasa, and K. Yabana, Phys. Rev. C 88, 014321 (2013).

[60] D. M. Brink, in Proceedings of the International School of Physics Enrico Fermi, Course 36, Varenna, edited by C. Bloch (Academic Press, New York, 1966).

[61] Y. Funaki, A. Tohsaki, H. Horiuchi, P. Schuck, and G. Röpke, Phys. Rev. C 67, 051306(R) (2003).

[62] M. Saraceno, P. Kramer, and F. Fernandez, Nucl. Phys. A 405, 88 (1983).

[63] J. Schnack and H. Feldmeier, Nucl. Phys. A 601, 181 (1996).

[64] A. Ono and H. Horiuchi, Phys. Rev. C 53, 2341 (1996).

[65] A. Ono and H. Horiuchi, Phys. Rev. C 53, 845 (1996).

[66] J. Schnack and H. Feldmeier, Phys. Lett. B 409, 6 (1997).

[67] Y. Sugawa and H. Horiuchi, Phys. Rev. C 60, 064607 (1999).

[68] T. Furuta and A. Ono, Phys. Rev. C 74, 014612 (2006).

[69] Y. Kanada-En'yo, M. Kimura, and A. Ono, Prog. Theor. Exp. Phys. 2012, 1A202 (2012).

[70] A. Volkov, Nucl. Phys. 74, 33 (1965).

[71] Y. Funaki, H. Horiuchi, and A. Tohsaki, Prog. Theor. Phys. 115, 115 (2006).

[72] F. Ajzenberg-Selove, Nucl. Phys. A 506, 1 (1990).

[73] M. Freer, I. Boztosun, C. A. Bremner, S. P. G. Chappell, R. L. Cowin, G. K. Dillon, B. R. Fulton, B. J. Greenhalgh, T. MunozBritton, M. P. Nicoli, W. D. M. Rae, S. M. Singer, N. Sparks, D. L. Watson, and D. C. Weisser, Phys. Rev. C 76, 034320 (2007).

[74] O. S. Kirsebom, M. Alcorta, M. J. G. Borge, M. Cubero, C. A. Diget, R. Dominguez-Reyes, L. M. Fraile, B. R. Fulton, H. O. U. Fynbo, S. Hyldegaard, B. Jonson, M. Madurga, A. Muñoz Martin, T. Nilsson, G. Nyman, A. Perea, K. Riisager, and O. Tengblad, Phys. Rev. C 81, 064313 (2010).

[75] M. Freer, M. Itoh, T. Kawabata, H. Fujita, H. Akimune, Z. Buthelezi, J. Carter, R. W. Fearick, S. V. Förtsch, M. Fujiwara, U. Garg, N. Hashimoto, K. Kawase, S. Kishi, T. Murakami, K. Nakanishi, Y. Nakatsugawa, B. K. Nayak, R. Neveling, S. Okumura, S. M. Perez, P. Papka, H. Sakaguchi, Y. Sasamoto, F. D. Smit, J. A. Swartz, H. Takeda, S. Terashima, M. Uchida, I. Usman, Y. Yasuda, M. Yosoi, and J. Zenihiro, Phys. Rev. C 86, 034320 (2012).

[76] D. J. Marín-Lámbarri, R. Bijker, M. Freer, M. Gai, T. Kokalova, D. J. Parker, and C. Wheldon, Phys. Rev. Lett. 113, 012502 (2014).

[77] P. Strehl, Z. Phys. A: Hadrons Nucl. 234, 416 (1970).

[78] T. Yamada, Y. Funaki, H. Horiuchi, K. Ikeda, and A. Tohsaki, Prog. Theor. Phys. 120, 1139 (2008).

[79] Y. Chiba, M. Kimura, and Y. Taniguchi, Phys. Rev. C 93, 034319 (2016).

[80] Y. Kanada-En'yo, Phys. Rev. C 93, 054307 (2016). 